\title{
Highlights of the issue 7, 2020
}

Published online: 8 July 2020

(C) Fondazione Società Italiana di Neurologia 2020

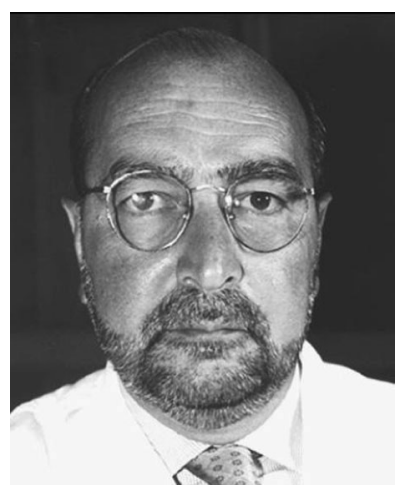

Antonio Federico

Editor-in-Chief

\section{COVID-19 Articles}

Real-time assessment of COVID-19 prevalence among multiple sclerosis patients: a multicenter European study Gloria Dalla Costa, Letizia Leocani, Xavier Montalban, Ana Isabel Guerrero,Per Soelberg Sørensen, Melinda Magyari, Richard J. B. Dobson, Nicholas Cummins, Vaibhav A. Narayan, Matthew Hotopf, Giancarlo Comi, on behalf of the RADAR-CNS consortium

(Italy, Spain, and Denmark, UK, Germany, USA)

https://link.springer.com/article/10.1007/s10072-020-04519-x

First motor seizure as presenting symptom of SARSCoV-2 infection

Antonio Fasano, Francesco Cavallieri, Elena Canali, Franco Valzania

(Italy)

https://link.springer.com/article/10.1007/s10072-020-04460-z

Keep "time is brain," even in the crisis of COVID-19

Doo Hyuk Kwon, Keun Tae Kim

(South Korea)

https://link.springer.com/article/10.1007/s10072-020-04483-6
New clinical manifestation of COVID-19 related Guillain-Barrè syndrome highly responsive to intravenous immunoglobulins: two Italian cases

Andrea Assini, Luana Benedetti, Silvia Di Maio, Erika Schirinzi, Massimo Del Sette

(Italy)

https://link.springer.com/article/10.1007/s10072-020-04484-5

So far so close: an insight into smart working and telehealth reorganization of a Language and Learning Disorders Service in Milan during COVID-19 pandemic

Daniela Sarti, Marinella De Salvatore, Stefania Gazzola, Chiara Pantaleoni, Elisa Granocchio (Italy)

https://link.springer.com/article/10.1007/s10072-020-04481-8

Brainstem involvement and respiratory failure in COVID-19

Fiore Manganelli, Maria Vargas, Aniello Iovino, Carmine Iacovazzo, Lucio Santoro, Giuseppe Servillo (Italy)

https://ink.springer.com/article/10.1007/s10072-020-04487-2

The neurological manifestations of COVID-19: a review article

Hamid Reza Niazkar, Behdad Zibaee, Ali Nasimi, Narjes Bahri

(Iran)

https://ink.springer.com/article/10.1007/s10072-020-04486-3

Stroke integrated care pathway during COVID-19 pandemic

Giovanni Frisullo, Antonio Giulio De Belvis, Giacomo Della Marca, Carmen Angioletti, Paolo Calabresi (Italy) 
https://link.springer.com/article/10.1007/s10072-020-04480-9

\section{Is it the right time for an infant screening for Duchenne muscular dystrophy?}

\section{Gian Luca Vita, Giuseppe Vita \\ (Italy)}

https://link.springer.com/article/10.1007/s10072-020-04307-7

Newborn screening (NBS) is an essential, preventive public health program for early identification of disorders whose early treatment can lead to significant reduction in morbidity and mortality . NBS for Duchenne muscular dystrophy (DMD) has been a controversial matter for many years, because of false positives, the lack of effective drugs and the need of more data about screening efficacy. The still high diagnostic delay of DMD and the current availability of drugs such as steroid, ataluren, eteplirsen, golodirsen and forthcoming new drugs, improving the clinical conditions if early started, make appropriate to begin a concrete discussion between stake holders to identify best practice for DMD screening. A twostep system CK/DNA screening programme is presented to be performed in male infants aged between 6 months and 42 months involving more than 30,000 male infants. Five to eight DMD subjects are believed to be diagnosed. The pilot project would give the opportunity to test in a small population the feasibility of an infant screening programme, which in the near future could be applicable to an entire country.

\section{Assessment of cutaneous axon-reflex responses to evaluate functional integrity of autonomic small nerve fibers}

Mido M.Hijazi, Sylvia J.Buchmann, Annahita Sedghi, Ben M. Illigens, Heinz Reichmann, Gabriele Schackert, Timo Siepmann (Germany, USA)

https://link.springer.com/article/10.1007/s10072-020-04293-w

Cutaneous autonomic small nerve fibers encompass unmyelinated $\mathrm{C}$-fibers and thinly myelinated $\mathrm{A} \delta$-fibers, which innervate dermal vessels (vasomotor fibers), sweat glands (sudomotor fibers), and hair follicles (pilomotor fibers). Analysis of their integrity can capture early pathology in autonomic neuropathies such as diabetic autonomic neuropathy or peripheral nerve inflammation due to infectious and autoimmune diseases. Furthermore, intraneural deposition of alpha-synuclein in synucleinopathies such as Parkinson's disease can lead to small fiber damage. Research indicated that detection and quantitative analysis of small fiber pathology might facilitate early diagnosis and initiation of treatment. While autonomic neuropathies show substantial etiopathogenetic heterogeneity, they have in common impaired functional integrity of small nerve fibers. This impairment can be evaluated by quantitative analysis of axonal responses to iontophoretic application of adrenergic or cholinergic agonists to the skin. The axon-reflex can be elicited in cholinergic sudomotor fibers to induce sweating and in cholinergic vasomotor fibers to induce vasodilation. Currently, only few techniques are available to quantify axon-reflex responses, the majority of which is limited by technical demands or lack of validated analysis protocols. Function of vasomotor small fibers can be analyzed using laser Doppler flowmetry, laser Doppler imaging, and laser speckle contrast imaging. Sudomotor function can be assessed using quantitative sudomotor axon-reflex test, silicone imprints, and quantitative direct and indirect testing of sudomotor function. More recent advancements include analysis of piloerection (goose bumps) following stimulation of adrenergicsmallfibersusingpilomotoraxon-reflex test. The AA provide a review of the current literature on axon-reflex tests in cutaneous autonomic small fibers

Eye tracking metrics to screen and assess cognitive impairment in patients with neurological disorders

Ling Tao, Quan Wang, Ding Liu, Jing Wang, Ziqing Zhu, Li Feng

(China)

https://ink.springer.com/article/10.1007/s10072-020-04310-y

Purpose of review Eye tracking is a powerful method to investigate the relationship between behavior and neural mechanisms. In recent years, eye movement analysis has been used in patients with neurological disorders to assess cognitive function. In this review, the AA explore the latest eye tracking researches in neurological disorders that are commonly associated with $\operatorname{cog}$ nitive deficits, specifically, amyotrophic lateral sclerosis (ALS), Alzheimer's disease (AD), Parkinson's disease (PD), multiple sclerosis (MS), and epilepsy. They focus on the application of ocular measures in these disorders, with the goal of understanding how eye tracking technology can be used in the clinical setting. Findings Eye tracking tasks (especially saccadic tasks) are of ten used as an adjunct to traditional scales for cognitive assessment. Eye tracking data confirmed that executive dysfunction is common in PD and ALS, whereas AD and MS are characterized by attention deficits. Research in evaluating cognitive function in epilepsy using eye tracking is still in its early stages, but this approach has shown advantages as a sensitive quantitative method with high temporal and spatial resolution. Summary Eye tracking technology can facilitate the assessment of cognitive impairment with higher temporal resolution and finer granularity than traditional cognitive assessment. Oculomotor data collected during cognitive tasks can provide insight into biological processes. Eye tracking provides a nonverbal and less cognitively demanding method of measuring disease progression in cognitively impaired patients. 
The effectiveness of various cytotherapeutic strategies for the treatment of ischemic stroke: a Bayesian network meta-analysis of randomized controlled trials

Kai Wang, Liangqun Rong, Xiue Wei, Qingxiu Zhang, Lijie Xiao

(China)

https://link.springer.com/article/10.1007/s10072-020-04312-w

Cytotherapy is a potential treatment for ischemic stroke (IS) patients but lacks uniform procedures. The AA aimed to assess the impact of the time of intervention, cell type, dose, and route of administration on the clinical effects by network metaanalysis. Methods They searched public electronic databases through July 7, 2019. Bayesian network meta-analyses were performed to compare differences among different cytotherapeutic strategies. Results Cytotherapy can significantly improve patients' activity of daily living according to the modified Rankin Scale (standard mean difference (SMD)-0.81; $95 \%$ confidence interval $(\mathrm{CI})-1.58,-0.03 ; \mathrm{p}=0.0417)$ and Barthel Index (SMD0.67; 95\% CI 0.05,1.30;p=0.036) results as well as improve neurological recovery (SMD-0.93;95\%CI $-1.29,-0.57 ; \mathrm{p}<0.001)$. Network meta-analysis showed that the intra-arterial injection of large amounts of mononuclear cells (NCs) or aldehyde dehydrogenase (ALDH)-positive cells was beneficial for improving patients' activity of daily living, while CD34+ cells through intracerebral injection had an advantage in the recovery of injured nerve function. Intravenous injection of mesenchymal stemcells (MSCs) or endothelial progenitor cells (EPCs) was beneficial in reducing mortality and serious adverse event (SAE) onset. Conclusions In the subacute stage, the intraarterial injection of NCs or ALDH cells improves patients' activity of daily living. Additionally, CD34+ cells through intracerebral injection had an advantage in the recovery of injured nerve function even in the chronic stage. Intravenous injection of MSCs or EPCs is a safety delivery route that can reduce mortality and SAE onset. However, further clinical studies are still needed to confirm these results.

\section{Eye movement changes in autosomal dominant spinocerebellar ataxias}

Francesca Rosini, Elena Pretegiani, Carla Battisti, Maria Teresa Dotti, Antonio Federico, Alessandra Rufa (Italy)

https://ink.springer.com/article/10.1007/s10072-020-04318-4

Oculomotor abnormalities are common findings in spinocerebellar ataxias (SCAs), a clinically heterogeneous group of neurodegenerative disorders with an autosomal dominant pattern of inheritance. Usually, cerebellar impairment accounts for most of the eye movement changes encountered; as the disease progresses, the involvement of extracerebellar structures typically seen in later stages may modify the oculomotor progression. However, ocular movement changes are rarely specific. In this regard, some important exceptions include the prominent slowing of horizontal eye movements in SCA2 and, to a lesser extent, in SCA3, SCA4, and SCA28, or the executive deficit in SCA2 and SCA17. The AA report the eye movement abnormalities and neurological pictures of SCAs through a review of the literature. Genetic and neuropathological/neuroimaging aspects are also briefly discussed. Overall, the findings reported indicate that oculomotor analysis could be of help in differential diagnosis among SCAs and contribute to clarify the role of brain structures, particularly the cerebellum, in oculomotor control.

\section{Clinico-diagnostic features of neuralgic amyotrophy in childhood}

Eleonora Rotondo, Noemi Pellegrino, Caterina Di Battista, Alessandro Graziosi, Vincenzo Di Stefano, Pasquale Striano (Italy)

https://ink.springer.com/article/10.1007/s10072-020-04314-8

Neuralgic amyotrophy (NA), even known as PersonageTurner's syndrome(PTS), is a neurologic condition, affecting the lower motor neurons of brachial plexus and/or individual nerves or nerve branches, characterized by pain, muscle weakness/atrophy, and sensory symptoms. NA has an acute/subacute onset, after an infection or vaccination ; it is more common in male and is rare in the pediatric population. The etiology remains uncertain, being considered heterogeneous and multifactorial. A severe acute neurologic pain around the shoulder girdle is the classic presenting symptom at onset. As the pain subsides, weakness and paresis develop. NA is usually unilateral, but sometimes, a subclinical contralateral limb involvement could be present and bilateral affection has been described. The diagnosis is clinical, through a comprehensive history and neurological examination. However, electrophysiological testing and imaging are critical, because there is no diagnostic test for PTS and it remains a diagnosis of exclusion. Upper brachial plexus peripheral involvement with weakness of periscapular and perihumeral muscles is the classic presentation, associated with electrophysiological evidence of denervation in the affected muscles. Imaging, laboratory, and genetic testing can be useful for the differential diagnosis. NA is in most cases a selflimiting condition, and it is characterized by good recovery. Treatment of NA usually involves a combination of corticosteroids, analgesics, immobilization, and physical therapy, even if limited data are available in children. Physiotherapy is required to maintain muscle strength 
Dysphasia is associated with diffusion-weighted MRI abnormalities in patients with transient neurological symptoms

Zejin Jia, Yangguang Song, Wenli Hu (China)

https://link.springer.com/article/10.1007/s10072-020-04258-z

Background The clinical characteristics of diffusionweighted imaging (DWI) abnormalities after transient neurological symptoms are of great significance for the early diagnosis and urgent intervention of transient ischemic attack (TIA). This study was aimed to investigate the clinical characteristics associated with acute DWI lesions in transient neurological symptoms. The AA retrospectively recruited 302 patients with transient neurological symptoms. According to DWI findings, they were divided into DWI positive and DWI negative group. The clinical characteristics and the TIA-related scores such as $A B C D 2, A B C D 3, A B C D 3 I$, Dawson score, and the Diagnosis of TIA (DOT) score were compared between the two groups. Logistic regression analysis and receiver operating characteristic curves were used to identify the independent factors and compare the predictive value of different TIA scores for acute DWI lesions. Results A total of 302 patients were enrolled in this study. The mean age was 61.8 years, and $67.2 \%$ were male. They found 89 (29.5\%) patients with DWI positivity. Logistic regression analysis showed the characteristic associated with DWI lesions was dysphasia (OR 2.226,95\% CI 1.220-4.062). The area under the curve for Dawson score and the DOT score was 0.610 (95\% CI $0.543-0.678)$ and 0.625 (95\% CI $0.559-0.691)$, respectively. They found that DWI lesions were detected in $29.5 \%$ of patients with transient neurological symptoms and were associated with dysphasia. Dawson score and DOT score could have a higher predictability of DWI lesions in patients with transient neurological symptoms.

\section{The influence of statins on the risk of post-stroke epilepsy}

\section{Bruno Kusznir Vitturi, Rubens José Gagliardi (Brazil)}

https://ink.springer.com/article/10.1007/s10072-020-04298-5

Currently, statins are widely used for secondary prevention of stroke due to their pleiotropic neuroprotective effects. Epilepsy is a common complication of cerebrovascular diseases. The purpose of this study was to evaluate the effect of statin therapy on the occurrence of post- stroke epilepsy (PSE). In this prospective cohort study, patients who suffered an ischemic stroke and without history of epilepsy before stroke were enrolled. At baseline, patients were classified according to the particularities of statin therapy. Statin use onset and adherence to treatment were registered as well. After a follow-up period of 1 year, we assessed the occurrence of seizures and PSE. Among the 477 patients included in our cohort, there were $91(19.1 \%)$ patients without statins, $160(33.5 \%)$ with simvastatin $20 \mathrm{mg}, 180(37.7 \%)$ with simvastatin $40 \mathrm{mg}$, and $46(9.6 \%)$ with high-potency statins. Overall, PSE emerged in 53(11.1\%) patients. PSE was significantly more prevalent among those who did not receive statins and those with lower doses of simvastatin. Acute onset of statin use was associated with reduced odds of having PSE. Conclusion Adequate treatment with statins after stroke may lower the risk of PSE

Elevated cerebrospinal fluid homocysteine is associated with blood-brain barrier disruption in amyotrophic lateral sclerosis patients

Yonghua $\mathrm{Wu}, \mathrm{Xu}$ Yang1 \& Xiaogang Li, Haining Wang, Tiancheng Wang

(China)

https://ink.springer.com/article/10.1007/s10072-020-04292-x

Homocysteine (Hcy) has been shown to be relevant in the pathogenesis of amyotrophic lateral sclerosis (ALS). Although the CSF Hcy changes were explored in patients with ALS previously, the outcomes were inconsistent, and the permeability of the blood-brain barrier(BBB) may involve in theprocess. The aim of this study was to investigate the relationship between concentration of Hcy and BBB integrity indicated by $\mathrm{CSF} / \mathrm{serum}$ albumin ratio (Qalb). Methods CSF and plasma/serum levels of Hcy, folate, and vitamin B12 and other biochemical biomarkers such as albumin, $\beta 2$ microglobulin, high sensitive-C reactive protein (hs-CRP), microalbumin, immunoglobulinG(IgG), IgM, IgA, and complement 3 and 4 were analyzed in all 31 ALS patients and 34 controls. Routine CSF analysis including cells/leukocytes count, total protein, glucose, and chlorides were also performed. Results CSF Hcy levels (0.50 $\pm 0.46 \mathrm{vs} 0.25 \pm 0.27 \mu \mathrm{mol} / \mathrm{L})$ and Qalb $(8.09 \pm 3.03 \mathrm{vs} 6.39$ \pm 2.21 ) were significantly higher in the ALS group than that in controls $(\mathrm{P}<0.05)$. The generalized linear mixed model analysis showed that the CSF Hcy was positively correlated with Qalb in ALS patients $(\mathrm{P}<0.05)$. Conclusions BBB permeability is increased in ALS patients. CSF Hcy level is associated with BBB integrity. Qalb is a significantly independent predisposing factor for CSF Hcy. 
The impact of white matter hyperintensities on speech perception

Arianna Di Stadio, Daniela Messineo, Massimo Ralli, Dalila Roccamatisi, Angela Musacchio, Giampietro Ricci, Antonio Greco

(Italy)

https://link.springer.com/article/10.1007/s10072-020-04295-8

The presence of white matter hyperintensities (WMHs) can impact on normal brain function by altering normal signal transmission and determining different symptoms. Aim To evaluate the relationship between the presence of brain WMHs and the scores of speech perception test (SPT) in a sample of normal-hearing patients under 70 years of age. Material and method Prospective study. One hundred eleven patients underwent audiological screening with pure tone audiometry (PTA), tympanometry, speech perception testing (SPT), and brain magnetic resonance imaging (MRI). T2 sequences were analyzed to identify the presence of WMH that, if identified, were scored using the Fazekas score. Statistical multiple regression analysis was performed to understand the relationship between PTA and SPT score; the Pearson's and Spearman's tests were used to evaluate the correlation between Fazekas scores and SPT. Chi-square test was used to analyze the difference between gender. The results of PTA were predictive of the SPT score. A negative statistically significant correlation (Spearman's, $\mathrm{p}=0.0001$; Pearson's, $\mathrm{p}<0.001$ ) was identified between the Fazekas score and the results of SPT. No statistically significant differences were identified in the correlation of WMH and SPT between males and females. Conclusion Multiple WMHs in the brain can worsen word recognition in patients with normal auditory threshold; this may be related to the impact that these lesions have on the memory ability. Spread of lesions into the brain might reduce the brain capacity to remember words, despite the sound is correctly perceived by the ear.

\section{Novel mutation of SCN9A gene causing generalized epilepsy with febrile seizures plus in a Chinese family}

Tian Zhang, Mingwu Chen, Angang Zhu, Xiaoguang Zhang, Tao Fang

(China)

https://link.springer.com/article/10.1007/s10072-020-04284-x

Generalized epilepsy with febrile seizures plus (GEFS+) is a complex familial epilepsy syndrome. It is mainly caused by mutations in SCN1A gene, encoding type 1 voltage-gated sodium channel $\alpha$-subunit (NaV1.1), and GABRA1 gene, encoding the $\alpha 1$ subunit of the $\gamma$-aminobutyric acid type A (GABAA) receptor, while seldom related with SCN9A gene, encoding the voltage-gated sodium channel NaV1.7. In this study, we investigated a Chinese family with an autosomal dominant form of GEFS+. DNA sequencing of the whole coding region revealed a novel heterozygous nucleotide substitution (c.5873A $>$ G) causing a missense mutation (p.Y1958C). This mutation was predicted to be deleterious by three different bioinformatics programs (The polyphen2, SIFT, and Mutation Taster). The finding reports a novel likely pathogenic SCN9AY1958C heterozygous mutation in a Chinese family with GEFS+ and provides additional supports that SCN9A variants may be associated with human epilepsies.

Publisher's note Springer Nature remains neutral with regard to jurisdictional claims in published maps and institutional affiliations. 